\title{
Effects of the COVID-19 pandemic on myocardial perfusion imaging for ischemic heart disease
}

\author{
Carmela Nappi ${ }^{1} \cdot$ Rosario Megna $^{2} \cdot$ Wanda Acampa $^{1} \cdot$ Roberta Assante $^{1} \cdot$ Emilia Zampella $^{1} \cdot$ Valeria Gaudieri $^{1}$. \\ Teresa Mannarino ${ }^{1} \cdot$ Roberta Green $^{1} \cdot$ Valeria Cantoni $^{1} \cdot$ Mario Petretta $^{3} \cdot$ Alberto Cuocolo $^{1}$ (D)
}

Received: 10 June 2020 / Accepted: 6 August 2020 / Published online: 11 August 2020

(C) Springer-Verlag GmbH Germany, part of Springer Nature 2020

\begin{abstract}
Purpose We assessed the effects of the COVID-19 pandemic on myocardial perfusion imaging (MPI) for ischemic heart disease during the lockdown imposed by the Italian Government.

Methods We retrospectively reviewed the number and the findings of stress single-photon emission computed tomography (SPECT)-MPI performed between February and May 2020 during the COVID-19 pandemic at the University of Napoli Federico II. The number and the findings of stress SPECT-MPI studies acquired in the corresponding months of the years 2017, 2018, and 2019 were also evaluated for direct comparison.

Results The number of stress SPECT-MPI studies performed during the COVID-19 pandemic $(n=123)$ was significantly lower $(P<0.0001)$ compared with the mean yearly number of procedures performed in the corresponding months of the years 2017, 2018, and $2019(n=413)$. Yet, the percentage of abnormal stress SPECT-MPI studies was similar $(P=0.65)$ during the pandemic (36\%) compared with the mean percentage value of the corresponding period of the years 2017, 2018, and 2019 (34\%).

Conclusion The number of stress SPECT-MPI studies was significantly reduced during the COVID-19 pandemic compared with the corresponding months of the previous 3 years. The lack of difference in the prevalence of abnormal SPECT-MPI studies between the two study periods strongly suggests that many patients with potentially abnormal imaging test have been missed during the pandemic.
\end{abstract}

Keywords COVID-19 $\cdot$ SARS-CoV-2 $\cdot$ SPECT-MPI $\cdot$ Ischemic heart disease $\cdot$ Southern Italy

\section{Introduction}

The recent COVID-19 emergency affected worldwide healthcare systems causing a rapid redefinition of the daily medical practice organization at every level [1-3]. To contain the spread of COVID-19 and to optimize hospital resources, all non-urgent diagnostic imaging studies, including nuclear cardiology procedures, have been postponed limiting the use

This article is part of the Topical Collection on Cardiology

Alberto Cuocolo

cuocolo@unina.it

1 Department of Advanced Biomedical Sciences, University Federico II, Naples, Italy

2 National Council of Research, Institute of Biostructure and Bioimaging, Naples, Italy

3 Department of Translational Medical Sciences, University Federico II, Naples, Italy of all available elective diagnostic imaging tools [4]. Suddenly, all the gained knowledge regarding the prognostic power of early diagnosis in all medicine fields have given the way to emergency measures with great concern about the clinical implications of primary prevention tool stoppage [5]. The reduction of timely diagnosis may have substantial consequences on diseases with high mortality rate, such as coronary artery disease (CAD) [6]. However, the real effect of this abrupt interruption has not yet been quantified.

Italy was among the first countries dramatically stricken by COVID-19 outbreak [7]. Therefore, the evaluation of the impact of containment measures against COVID-19 spread on the non-COVID-19 diseases in Italy may measure the magnitude of the effect on larger scale. Piccolo et al. [8] recently investigated the association between the outbreak of COVID19 and percutaneous coronary intervention (PCI) rates for acute coronary syndrome (ACS) in the Campania region, accounting for about $10 \%$ of the entire Italian population. They found evidence that the outbreak of COVID-19 was 
associated with a decline by $32 \%$ in the number of PCI for ACS suggesting two potential explanations. If on one hand chest pain, which is the most frightening sign who lead patients to emergency room, might be underestimated due to the COVID-19 fear, on the other, physical activity that might trigger an ACS has significantly decreased during lockdown. The aim of this study was to assess the impact of the COVID19 pandemic on stress single-photon emission computed tomography (SPECT) myocardial perfusion imaging (MPI) work-up for ischemic heart disease during the lockdown imposed by the Italian Government [7]. For this purpose, we reviewed the data of stress SPECT-MPI performed between February and May 2020, in comparison with the corresponding months of the years 2017, 2018, and 2019.

\section{Methods}

\section{Study protocol}

All patients included in the study were part of ongoing prospective dedicated database [9]. The study was approved by the Ethical Committee "Carlo Romano," University of Naples Federico II, and all patients were informed and signed a written consent to participate to this study.

\section{Clinical definitions}

As part of the baseline examination, clinical teams collected information on traditional cardiovascular risk factors, including age, gender, chest pain, smoking history, family history of CAD, diabetes, hypertension, and known CAD. Chest pain was classified according to the American College of Cardiology/American Heart Association 2002 guideline update on exercise testing [10]. Patients were considered as having diabetes if they were receiving treatment with oral hypoglycemic drugs or insulin. A family history of premature CAD was defined as a diagnosis of CAD in a first-degree relative prior to or at 55 years of age. Hypertension was defined as a blood pressure $>140 / 90 \mathrm{mmHg}$ or use of antihypertensive medication. Hyperlipidemia was defined as total cholesterol level $>6.2 \mathrm{mmol} / \mathrm{L}$ or treatment with cholesterol lowering medication. Smoking history was defined as prior or current tobacco use. A patient was considered to have known CAD at the time of imaging based on a provided history of previously diagnosed atherosclerotic coronary disease, history of myocardial infarction (chest pain or equivalent symptom complex, positive cardiac biomarkers, or typical electrocardiographic changes), history of percutaneous coronary intervention, or history of coronary artery bypass grafting.

\section{Myocardial perfusion imaging}

Patients underwent stress-optional rest ${ }^{99 \mathrm{~m}} \mathrm{Tc}$ sestamibigated stress SPECT-MPI by physical exercise or dipyridamole stress test, according to the recommendations of the European Association of Nuclear Medicine [11]. During the pandemic, pharmacological stress was preferred to exercise stress test and criteria to prioritize patients were implemented, according to the recommendations of the American Society of Nuclear Cardiology and Society of Nuclear Medicine and Molecular Imaging [4]. Decisions were made based on clinical judgment on expected benefit to guide management, on the urgency of the test, and in discussion with the referring clinician. SPECT-MPI was performed as scheduled: (1) for evaluation of ischemia in moderate- to high-risk patients considered for urgent coronary revascularization, (2) in patients with intermediate pretest CAD likelihood or when suspicion of CAD was high but coronary angiography had greater risk, and (3) for evaluation of ischemia in moderate- to high-risk patients in whom surgical procedure was urgent and revascularization was an option. On the other hand, the test was postponed by 2-4 months for evaluation of ischemia follow-up when there was no urgent revascularization plan.

At peak exercise, or 4 min after completion of dipyridamole infusion, a bolus of $155 \mathrm{MBq}$ of ${ }^{99 \mathrm{~m}} \mathrm{Tc}$ sestamibi was intravenously injected $[12,13]$. For stress imaging studies, recordings were obtained by cadmiumzinc telluride (CZT)-SPECT (D-SPECT, Spectrum Dynamics, Caesarea, Israel) system using 9 pixilated CZT crystal detector columns mounted vertically spanning a $90^{\circ}$ geometry [14]. Each of the columns consists of 1024 $(16 \times 64) 5-\mathrm{mm}$ thick CZT crystal elements $(2.46 \times$ $2.46 \mathrm{~mm}$ ). Square hole tungsten collimators are fitted to each of the detectors, which are shorter than conventional low-energy, high-resolution collimators, yielding significantly better geometric speed. Data were acquired focusing on the heart by the detectors rotating in synchrony and saved in list mode. Images were obtained with the patient in a semi-recumbent position. A 10-s pre-scan acquisition was performed to identify the location of the heart and to set the angle limits of scanning for each detector (region of interest-centric scanning). Using the myocardial count rate from the pre-scan acquisition, the time per projection was set to target the recording of 1000 myocardial kcounts. For rest-optional images, a second intravenous bolus of $370 \mathrm{MBq}$ of ${ }^{99 \mathrm{~m}} \mathrm{Tc}$-sestamibi was injected followed by rest imaging recording. The duration of the scans was less than $10 \mathrm{~min}$ for stress and $4 \mathrm{~min}$ for rest. Summed and gated projections were reconstructed with an iterative maximum likelihood expectation maximization algorithm using 7 and 4 iterations, respectively [15]. 
Fig. 1 Number of stress SPECTMPI procedures performed during COVID-19 pandemic and during corresponding months of the years 2017,2018 , and $2019(P$ for trend $<0.0001$ )

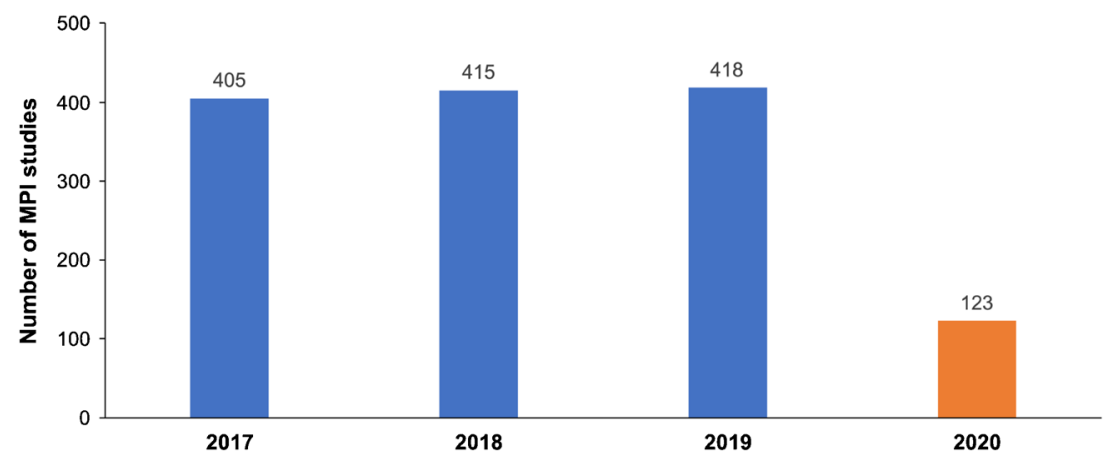

\section{Imaging interpretation}

An automated software program (e-soft, 2.5, QGS/QPS, Cedars-Sinai Medical Center, Los Angeles, CA) was used to calculate the scores incorporating both the extent and severity of perfusion defects, using standardized segmentation of 17 myocardial regions [16]. The summed stress score (SSS), representing the total abnormal myocardium (i.e., necrotic and ischemic tissue), was obtained by adding the scores of the 17 segments of the stress images. A SSS > 3 was considered abnormal [17]. A similar procedure was applied to the resting images to calculate the summed rest score and the summed difference score. Total perfusion defect reflecting a combination of both severity and extent of myocardial defect was also calculated and categorized according to the presence of $\geq 10 \%$ of abnormal myocardium.

\section{Statistical analysis}

Continuous data are expressed as mean \pm standard deviation and categorical data as percentage. Student's $t$ test and $\chi^{2}$ test were used to compare the differences in continuous and categorical variables, respectively. Poisson regression was used to compare the number of SPECT-MPI test performed during the lockdown and those during the corresponding time interval of the previous 3 years. Multivariable logistic regression analysis for predicting abnormal SPECT-MPI was performed for lockdown period and corresponding period of previous 3 years. Two-tailed $P$ values $<0.05$ were considered significant. Statistical analysis was performed with $\mathrm{R}$ software version
3.6.3 (R Foundation for Statistical Computing, Vienna, Austria).

\section{Results}

A total of 1361 stress SPECT-MPI were considered, including all tests performed during the pandemic between February and May 2020 and those performed in the corresponding months of the prior 3 years $(2017,2018$, and 2019).

The number of stress SPECT-MPI studies performed during the pandemic $(n=123)$ was significantly lower $(P<0.0001)$ compared with the mean number of procedures in the corresponding months of the years 2017, 2018, and 2019 ( $n=413$ ) (Fig. 1). However, the percentage of abnormal stress SPECT-MPI studies was similar $(P=0.65)$ during the pandemic $(36 \%)$ compared with mean percentage value of the corresponding months of the years 2017, 2018, and 2019 (34\%) (Fig. 2). Of note, given the mean number of $139 \mathrm{ab}-$ normal stress SPECT-MPI in the previous 3 years and the number of 44 abnormal tests during the pandemic, there was a $68 \%$ of abnormal test missed. Baseline characteristics of the entire population according to the year of SPECT-MPI execution are reported on Table 1 . Most of the considered variables were comparable between the two study periods.

Characteristics of overall population are also described according to stress SPECT-MPI results during COVID-19 pandemic and during the corresponding months of 2017-2019 (Table 2). In both study periods, the percentage of patients with hyperlipidemia and known CAD was higher in patients
Fig. 2 Prevalence of abnormal stress SPECT-MPI studies during COVID-19 pandemic and during the corresponding months of the years 2017, 2018, and 2019. The percentage of abnormal stress SPECT-MPI studies was similar $(P=0.65)$

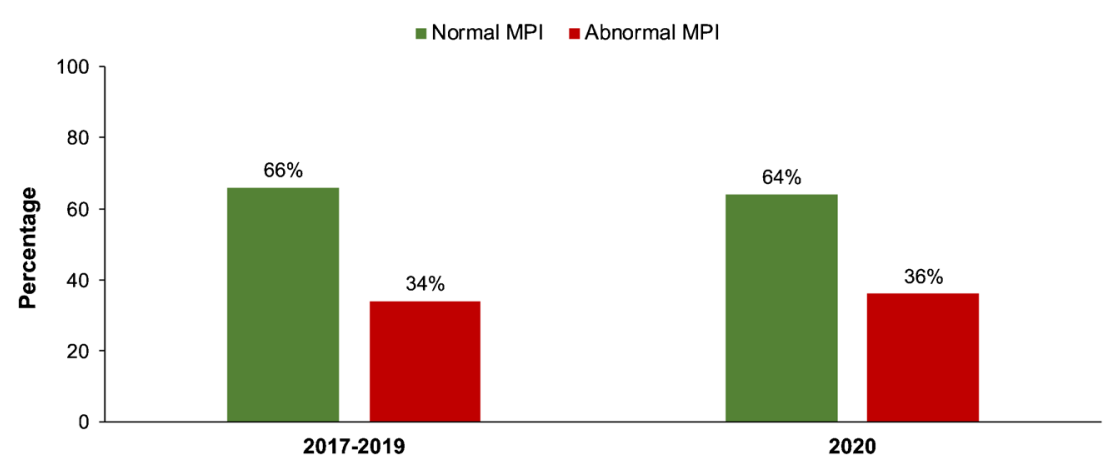


Table 1 Baseline characteristics of overall population according to the year of MPI execution

\begin{tabular}{lcccc}
\hline & All $(n=1361)$ & $2020(n=123)$ & $2017-2019(n=1238)$ & $P$ value \\
\hline Age (years) & $64 \pm 11$ & $64 \pm 10$ & $64 \pm 11$ & 0.14 \\
Male gender, $n(\%)$ & $439(32)$ & $29(24)$ & $410(33)$ & $<.05$ \\
Body mass index $\left(\mathrm{kg} / \mathrm{m}^{2}\right)$ & $29 \pm 5$ & $29 \pm 5$ & $29 \pm 6$ & 0.43 \\
Chest pain, $n(\%)$ & $468(34)$ & $37(30)$ & $431(35)$ & 0.29 \\
Diabetes, $n(\%)$ & $437(32)$ & $47(38)$ & $390(32)$ & 0.12 \\
Dyspnea, $n(\%)$ & $624(46)$ & $49(40)$ & $575(46)$ & 0.16 \\
Family history, $n(\%)$ & $729(54)$ & $60(49)$ & $669(54)$ & 0.26 \\
Hypertension, $n(\%)$ & $1070(79)$ & $102(83)$ & $968(78)$ & 0.22 \\
Hyperlipidemia, $n(\%)$ & $949(70)$ & $92(75)$ & $857(69)$ & 0.20 \\
Smoking, $n(\%)$ & $416(31)$ & $26(21)$ & $390(32)$ & 0.05 \\
Known CAD, $n(\%)$ & $593(44)$ & $52(42)$ & $541(44)$ & $<.76$ \\
Stress type & & & & \\
Physical exercise test, $n(\%)$ & $519(38)$ & $11(9)$ & $508(41)$ & $730(59)$ \\
Pharmacological test, $n(\%)$ & $842(62)$ & $112(91)$ & $418(34)$ & 0.65 \\
Abnormal MPI, $n(\%)$ & $462(34)$ & $44(36)$ & $219(52)$ & 0.26 \\
Total perfusion defect $>10 \%$ & $246(53)$ & $27(61)$ &
\end{tabular}

Values are expressed as mean value \pm standard deviation or as number (percentage) of subjects. MPI myocardial perfusion imaging, $C A D$ coronary artery disease with abnormal compared with those with normal stress SPECT-MPI findings, while during 2017-2019, patients with abnormal SPECT-MPI were older $(P<0.05)$ and had higher prevalence of diabetes $(P<0.005)$ and hypertension $(P<0.001)$.

At multivariable logistic regression analysis (Table 3 ), hypertension $(P<0.05)$ and known CAD $(P<0.001)$ were significantly associated with abnormal stress SPECT-MPI during both COVID-19 pandemic and the corresponding months of the previous 3 years. No significant interactions among risk factors were found. During the 2017-2019 period, also male gender $(P<0.001)$ and diabetes $(P<0.05)$ were associated with abnormal stress SPECT-MPI.

\section{Discussion}

The results of this study show that, due to the restrictive medical measures for COVID-19 emergency, the number of stress SPECT-MPI procedures was significantly reduced during the

Table 2 Clinical characteristics of overall population according to MPI results during COVID-19 emergency and during 2017-2019 3-year's corresponding months

\begin{tabular}{|c|c|c|c|c|c|c|}
\hline & \multicolumn{3}{|l|}{2020} & \multicolumn{3}{|l|}{ 2017-2019 } \\
\hline & Normal $(n=79)$ & Abnormal $(n=44)$ & $P$ value & Normal $(n=820)$ & Abnormal $(n=418)$ & $P$ value \\
\hline Age (years) & $64 \pm 11$ & $64 \pm 8$ & 0.77 & $63 \pm 11$ & $65 \pm 11$ & $<.05$ \\
\hline Male gender, $n(\%)$ & $23(38)$ & $6(43)$ & 0.05 & $337(41)$ & $73(17)$ & $<.001$ \\
\hline Body mass index $\left(\mathrm{kg} / \mathrm{m}^{2}\right)$ & $28.9 \pm 4.9$ & $28.1 \pm 4.4$ & 0.38 & $29.1 \pm 6.1$ & $27.9 \pm 4.2$ & $<.001$ \\
\hline Chest pain, $n(\%)$ & $22(38)$ & $15(43)$ & 0.47 & $308(38)$ & $123(29)$ & $<.005$ \\
\hline Diabetes, $n(\%)$ & $28(19)$ & $19(36)$ & 0.40 & $234(29)$ & $156(37)$ & $<.005$ \\
\hline Dyspnea, $n(\%)$ & $29(22)$ & $20(25)$ & 0.34 & $375(46)$ & $200(48)$ & 0.48 \\
\hline Family history, $n(\%)$ & $36(48)$ & $24(41)$ & 0.34 & $446(54)$ & $223(53)$ & 0.73 \\
\hline Hypertension, $n(\%)$ & $66(62)$ & $36(76)$ & 0.81 & $614(75)$ & $354(85)$ & $<.001$ \\
\hline Hyperlipidemia, $n(\%)$ & $54(55)$ & $38(57)$ & $<.05$ & $540(66)$ & $317(76)$ & $<.001$ \\
\hline Smoking, $n(\%)$ & $15(55)$ & $11(57)$ & 0.43 & $258(31)$ & $132(32)$ & 0.97 \\
\hline Known CAD $n(\%)$ & $21(55)$ & $31(57)$ & $<.001$ & $224(27)$ & $317(76)$ & $<.001$ \\
\hline
\end{tabular}

Values are expressed as mean value \pm standard deviation or as number (percentage) of subjects. $M P I$ myocardial perfusion imaging, $C A D$ coronary artery disease 
Table 3 Multivariable logistic regression analysis with abnormal MPI as dependent variable during COVID-19 emergency and during 2017-2019 3-year's corresponding months

\begin{tabular}{|c|c|c|c|c|c|c|}
\hline & \multicolumn{3}{|l|}{2020} & \multicolumn{3}{|l|}{ 2017-2019 } \\
\hline & $\beta$ coefficient & $\mathrm{SE}$ & $P$ value & $\beta$ coefficient & SE & $P$ value \\
\hline Age & 0.005 & 0.023 & 0.834 & -0.004 & 0.007 & 0.60 \\
\hline Male gender & 0.162 & 0.639 & 0.799 & 0.671 & 0.166 & $<.001$ \\
\hline Body mass index & -0.013 & 0.049 & 0.785 & -0.024 & 0.014 & 0.08 \\
\hline Chest pain & 0.542 & 0.508 & 0.286 & -0.264 & 0.149 & 0.08 \\
\hline Diabetes & 0.565 & 0.466 & 0.225 & 0.310 & 0.148 & $<.05$ \\
\hline Family history & 0.180 & 0.454 & 0.692 & -0.163 & 0.150 & 0.28 \\
\hline Dyspnea & 0.362 & 0.382 & 0.343 & -0.085 & 0.120 & 0.48 \\
\hline Hypertension & -1.573 & 0.709 & $<.05$ & 0.376 & 0.196 & $<.05$ \\
\hline Hyperlipidemia & 0.822 & 0.621 & 0.186 & -0.239 & 0.167 & 0.15 \\
\hline Smoking & 0.619 & 0.533 & 0.245 & -0.102 & 0.152 & 0.50 \\
\hline Known CAD & 0.137 & 0.541 & $<.001$ & 1.994 & 0.154 & $<.001$ \\
\hline
\end{tabular}

$S E$ standard error, $M P I$ myocardial perfusion imaging, $C A D$ coronary artery disease pandemic compared with the corresponding months of the previous 3 years (i.e., 2017, 2018, and 2019). However, the proportion of abnormal stress SPECT-MPI was not different between the two study periods. This finding suggests that a significant number of patients, who would have performed MPI test with abnormal results, have been missed during the COVID-19 pandemic. Given the mean number of 139 abnormal stress SPECT-MPI detected at our institution in the previous 3 years during the time range corresponding to the COVID-19 pandemic, and given the 44 abnormal tests observed during COVID-19 outbreak, it can be hypothesized that there is a $68 \%$ of abnormal test missed.

Piccolo et al. [8] recently investigated the association between the outbreak of COVID-19 and primary PCI rates due to ACS in the Campania region. They found evidence that the outbreak of COVID-19 was associated with a decline by $32 \%$ in the number of PCI due to ACS. Data presented by Piccolo and co-workers [7] refer to the same region from which the patients belonging to this department come from. Even more concern may rise looking at reduced admissions for acute myocardial infarction during the COVID-19 pandemic not only in Campania region but also across Italy, with a parallel increase in fatality and complication rates [18].

Our data also show that while patients with abnormal stress SPECT-MPI findings during the previous years 2017-2019 were older with higher proportion of diabetes and hypertension compared with those with normal imaging results, during pandemic COVID-19 outbreak, there was no difference between patients with normal and abnormal tests as regards to these clinical risk factors. This finding is not surprising given the high concern for vulnerability to COVID-19 in frail patients such as elderly subjects with hypertension and diabetes $[19,20]$. Hence, it is reasonable that regarding frail population, the anxiety to become exposed to COVID-19 contagion while performing medical controls and diagnostic procedures has dominated the will to monitor CAD development on both sides of therapeutic alliance, from patient side through media communication and from health system side who was focused on relocation resources on COVID-19 pandemic management.

The lesson we learned from this global experience may be useful to improve nuclear medicine laboratories' daily activities. Such implementation should have been put into practice as standard protocol rather than only during potential new emergencies. This is the only way to assure high-quality healthcare performances in terms of numbers and therapeutic strategies regardless specific contingencies without sacrificing medical assistance in favor of emergency needs. Protection of healthcare personnel, with personal protective equipment use and strict hand and laboratory hygiene, should be always recommended. To minimize contact, all information regarding the performing test, clinical history data, and consent to undertake the test should be provided prior to the patients' onsite arrival. On the other hand, patient's compliance should be always assured with a brief and safe interview before test. Regarding the choice of imaging protocol during the pandemic, this aspect should take into account only the diagnostic and prognostic evidences provided by literature as regards to the maximum benefit of patients from the type of test performed preferring 1-day-pharmacological stress-first SPECT-MPI protocols to optimize not only radioactive dose exposure but also the time of patient staying in hospital facilities. Patient selection for SPECT-MPI should be based on clinical judgment on expected benefit to guide management, on the urgency of the test, and in discussion with the referring clinician.

There are numerous physical and social scars from the pandemic COVID-19 emergency that humanity is called to manage. Among those directly linked to COVID-19 disease, 
human beings need to be aware and fully prepared to deal with those less evident when all the lights were on SARS-COV2 virus. There is a concrete risk to have gone back to the preprimary prevention era, when diagnosis and treatments were reached too late. Thus, there is an urgent need to restore mutual trust in all hospital facilities as places of care rather than virus spread centers to avoid a further, long-term and no less dangerous effect of COVID-19 outbreak on non-COVID-19 patients saved from the virus storm but not from the darkness of carelessness.

\section{Conclusion}

The number of stress SPECT-MPI studies was significantly reduced during the COVID-19 pandemic compared with the corresponding months of the previous 3 years. The lack of difference in the prevalence of abnormal stress SPECT-MPI studies between the two study periods strongly suggests that many patients with potentially abnormal test have been missed during the pandemic, in particular elderly subjects with hypertension and diabetes.

Authors' contribution $\mathrm{CN}, \mathrm{RM}, \mathrm{MP}$, and AC conceptualized the paper; WA, RA, EG, TM, RG, and VC evaluated and reported the imaging findings; $\mathrm{CN}, \mathrm{RM}, \mathrm{MP}$, and $\mathrm{AC}$ drafted the manuscript; and all the authors revised and commented on the paper and approved the final version of the manuscript.

\section{Compliance with ethical standards}

Conflict of interest The authors declare that they have no conflict of interest.

Ethical approval All procedures performed in this study involving human participants were in accordance with the ethical standards of the institutional and/or national research committee and with the 1964 Helsinki declaration and its later amendments or comparable ethical standards.

Informed consent Informed consent was obtained from all individual participants included in the study.

\section{References}

1. Nacoti M, Ciocca A, Giupponi A, Brambillasca P, Lussana F, Pisano M, et al. At the epicenter of the Covid-19 pandemic and humanitarian crises in Italy: changing perspectives on preparation and mitigation [published online ahead of print, 2020 March 21]. NEJM Catalyst. 2020. https://doi.org/10.1056/CAT.20.0080.

2. Stempniak M. Mednax sees 'meaningful' decline in radiology volume during pandemic, revises revenue forecasts. Radiology Business. 2020. https://www.radiologybusiness.com/topics/ healthcare-economics/mednax-radiology-volume-coronaviruscovid-19-imaging.
3. Cavallo JJ, Forman HP. The economic impact of the COVID-19 pandemic on radiology practices. Radiology. 2020. https://doi.org/ 10.1148/radiol.2020201495.

4. Skali H, Murthy VL, Paez D, Choi EM, Keng FYJ, Iain MA, et al. Guidance and best practices for reestablishment of non-emergent care in nuclear cardiology laboratories during the coronavirus disease 2019 (COVID-19) pandemic: an information statement from ASNC, IAEA, and SNMMI. J Nucl Med. 2020. https://doi.org/10. 2967/jnumed.120.251355.

5. Nappi C, Nicolai E, Daniele S, Acampa W, Gaudieri V, Assante R, et al. Long-term prognostic value of coronary artery calcium scanning, coronary computed tomographic angiography and stress myocardial perfusion imaging in patients with suspected coronary artery disease. J Nucl Cardiol. 2018;25:833-41.

6. Wijns W, Naber CK. Reperfusion delay in patients with high-risk ST-segment elevation myocardial infarction: every minute counts, much more than suspected. Eur Heart J. 2018;39:1075-7.

7. Law Decree n. 6, issued on 23 February 2020. Available from the official source of knowledge of the rules in force in Italy. https:// www.gazzettaufficiale.it/eli/id/2020/02/23/20G00020/sg.

8. Piccolo R, Bruzzese D, Mauro C, Aloia A, Baldi C, Boccalatte M, et al. Population trends in rates of percutaneous coronary revascularization for acute coronary syndromes associated with the COVID-19 outbreak [published online ahead of print, 2020 Apr 30]. Circulation. 2020. https://doi.org/10.1161/ CIRCULATIONAHA.120.047457.

9. Megna R, Petretta M, Alfano B, Cantoni V, Green R, Daniele S, et al. A new relational database including clinical data and myocardial perfusion imaging findings in coronary artery disease. Curr Med Imaging Rev. 2019;15:661-71.

10. Gibbons RJ, Balady GJ, Bricker JT, Chaitman BR, Fletcher GF, Froelicher VF, et al. ACC/AHA 2002 guideline update for exercise testing: summary article: a report of the American College of Cardiology/American Heart Association Task Force on Practice Guidelines (Committee to Update the 1997 Exercise Testing Guidelines). Circulation. 2002;106:1883-92.

11. Verberne HJ, Acampa W, Anagnostopoulos C, Ballinger J, Bengel $\mathrm{F}$, De Bondt $\mathrm{P}$, et al. EANM procedural guidelines for radionuclide myocardial perfusion imaging with SPECT and SPECT/CT: 2015 revision. Eur J Nucl Med Mol Imaging. 2015;2015:1929-40.

12. Acampa W, Zampella E, Assante R, Genova A, De Simini G, Mannarino T, et al. Quantification of myocardial perfusion reserve by CZT-SPECT: a head to head comparison with 82Rubidium PET imaging [published online ahead of print, 2020 May 7]. J Nucl Cardiol. 2020. https://doi.org/10.1007/s12350-020-02129-w.

13. Fletcher GF, Ades PA, Kligfield P, Arena R, Balady GJ, Bittner VA, et al. Exercise standards for testing and training: a scientific statement from the American Heart Association. Circulation. 2013;128:873-934.

14. Gambhir SS, Berman DS, Ziffer J, Nagler M, Sandler M, Patton J, et al. A novel high sensitivity rapid acquisition single photon cardiac imaging camera. J Nucl Med. 2009;50:635-43.

15. Mannarino T, Assante R, Ricciardi C, Zampella E, Nappi C, Gaudieri V, et al. Head-to-head comparison of diagnostic accuracy of stress-only myocardial perfusion imaging with conventional and cadmium-zinc telluride single-photon emission computed tomography in women with suspected coronary artery disease [published online ahead of print, 2020 Jun 20]. J Nucl Cardiol. 2019. https:// doi.org/10.1007/s12350-019-01789-7.

16. Germano G, Kavanagh PB, Waechter P, Areeda J, Van Kriekinge $\mathrm{S}$, Sharir T, et al. A new algorithm for the quantitation of myocardial perfusion SPECT. I: technical principles and reproducibility. J Nucl Med. 2000;41:712-9.

17. Nappi C, Gaudieri V, Acampa W, Assante R, Zampella E, Mainolfi $\mathrm{CG}$, et al. Comparison of left ventricular shape by gated SPECT imaging in diabetic and nondiabetic patients with normal 
myocardial perfusion: a propensity score analysis. J Nucl Cardiol. 2018;25:394-403.

18. De Rosa S, Spaccarotella C, Basso C, Calabrò MP, Curcio A, Filardi PP, et al. Reduction of hospitalizations for myocardial infarction in Italy in the COVID-19 era. Eur Heart J. 2020;41:2083-8.

19. Fang L, Karakiulakis G, Roth M. Are patients with hypertension and diabetes mellitus at increased risk for COVID-19 infection? Lancet Respir Med. 2020;8:e21.
20. Sinclair AJ, Abdelhafiz AH. Age, frailty and diabetes - triple jeopardy for vulnerability to COVID-19 infection. EClinicalMedicine. 2020;22:100343.

Publisher's note Springer Nature remains neutral with regard to jurisdictional claims in published maps and institutional affiliations. 